\title{
Novel Algorithm in Activation Process of GPON Networks
}

\author{
Tomas Horvath, Petr Munster, Michal Jurcik, and Miloslav Filka
}

\begin{abstract}
Passive optical networks are dominating access networks around the world due to its their economical aspect, bandwidth, and penetration. It is necessary to deal with specification of these networks. The article deals with a transmission convergence layer of a Gigabit passive optical network, especially the activation process for ONUs. When the blackout affects all ONUs in the network, all ONUs need to be resynchronized with OLT but the current algorithm affects that for higher value of ONUs synchronization after a couple of minutes. We proposed a novel algorithm for an ONU activation time with the same frame structure and state machine, which are defined in the main specification. We verified the proposed algorithm by simulation and compare results for the current and novel algorithms. Our results confirm that it is possible to achieve the better time up to nineteen times lower synchronization time in comparison with the current algorithm.
\end{abstract}

Index Terms-GPON, Transmission Convergence layer, simulation, algorithm.

\section{INTRODUCTION}

Nowadays, the PONs (Passive Optical Networks) are widely used around the world. The basic classification of the passive optical networks is in accordance with a transferring protocol: Ethernet frame or ITU (International Telecommunication Union) encapsulation method. The PONs according to IEEE (Institute of Electrical and Electronics Engineers) are dominating in Asia, especially the EPON (Ethernet PON) standard. However, in this article we deal only with the ITU standards, particularly with the GPON (Gigabit PON) approved by ITU in 2003 standard because this standard is dominating in the Europe.

The European Union requires at least $30 \mathrm{Mb} / \mathrm{s}$ of the bandwidth to each household until 2020. It is necessary to deal with the gigabit passive optical networks because this technology is mature and widely used in the Czech Republic by ISPs (Internet Services Providers). However, ISPs can use newer technology called XG-PON (Next Generation PON) they use the GPON technology due to its mature. Fig. 1 shows a roadmap of the ITU standards for PONs.

The principle of the PON networks can be explained by the following terms: ONU (Optical Network Unit), OLT (Optical Line Termination) and ODN (Optical Distribution Network).

Manuscript received September 25, 2015; revised December 31, 2015.

Research described in this paper was financed by the National Sustainability Program under grantLO1401, and SIX CZ.1.05/2.1.00/03.0072. For the research, infrastructure of the SIX Center was used.

Authors are with Department of Telecommunications, Brno University of Technology, Brno, Czech Republic (E-mails: horvath@feec.vutbr.cz, munster@feec.vutbr.cz,xjurci03@stud.feec.vutbr.cz, filka@feec.vutbr.cz).

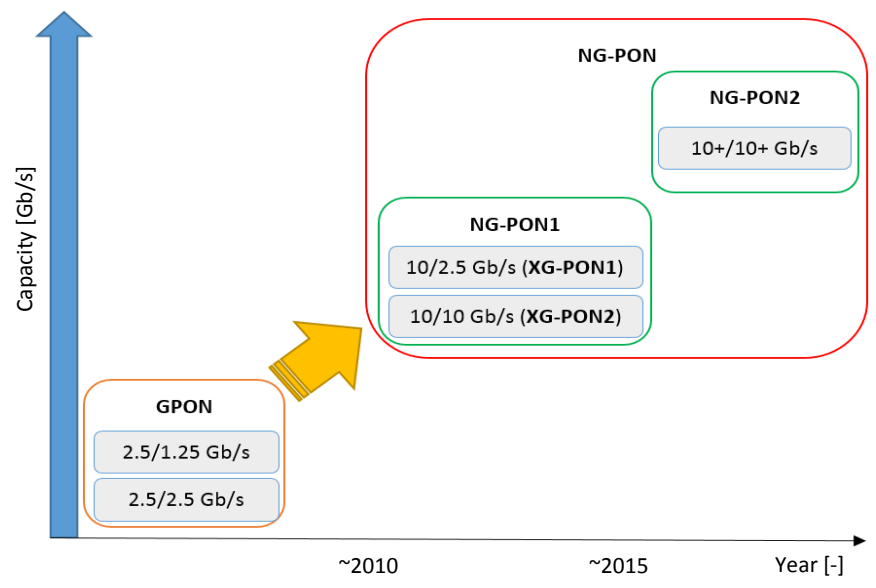

Fig. 1. ITU standards roadmap [1].

In general, the OLT is located in the CO (Central Office) and ONU is located in the customer part of the network, everything else between OLT and ONU is called ODN (optical fibres, splicing, splitters, and connectors). In general, the communication slots are allocated by the TDM (Time Division Multiplex) for GPON but newer standards are based on combination of the TDM and WDM (Wavelength Division Multiplex), especially XG-PON and NG-PON2 (Next Generation PON second generation). The description of two latest standards is out of the scope of this article.

In the following part, we describe the GPON in more details. The GPON standard has specification of physical and TC (Transmission Convergence) layers. The physical layer is specified by the ITU-T G.984.1 document where the most important part for the ODN is described. In general, the document specifies the split ratio, bandwidth, wavelengths etc. It is necessary to define two types of the GPON networks: standard and long reach networks. We deal with a typical network with ODN length of $20 \mathrm{~km}$, split ratio at least 1:64 (up to $1: 128)$, and $1480-1500 \mathrm{~nm}$ for downstream and $1260-1360 \mathrm{~nm}$ for upstream wavelengths, specified by [2]. Furthermore, the bandwidth can be allocated in symmetric or asymmetric mode: symmetric mode uses 1.24416 or $2.48832 \mathrm{~Gb} / \mathrm{s}$, asymmetric mode defines 1.24416 or $2.48832 \mathrm{~Gb} / \mathrm{s}$ for downstream and $0.15552,0.62208$, and $1.24416 \mathrm{~Gb} / \mathrm{s}$ for upstream direction. A specification of the TC layer is more complicated but the most important part is an initialization process for each ONU in ODN. Specification of the TC layer will be described later in the chapter. 
The current algorithm of ONU activation process allows to register only the single ONU in one period. That is the reason, why the last ONU can be connected after tens of seconds due to actual behaviour of algorithm. We proposed a novel algorithm for ONU activation process. The main contribution of this paper is the algorithm which reduces the total time of activation process with the current frame structure and state machine.

The rest of this paper is structured as follows. Section 2 gives an overview of some other related works. Section 3 describes the GPON technology and ONU activation process. Section 4 shows a designed model of current and novel algorithms. Section 5 discusses the achieved results and Section 6 concludes the paper.

\section{RELATED WORKS}

Since 1995 many standards of passive optical networks have been developed. In GPON, the downstream is transferred via broadcast for each ONU, only ONU with the same identifiers is able to read the frame. The upstream direction is transmitted via unicast. There are many works which deal with the physical and TC layers of the GPON.

The authors in [3] describe the TC layer for the XG-PON networks. They focus on the comparison of frame structures for the GPON and XG-PON from the point of view of upstream and downstream efficiency. The results presented XGPON networks as much more efficiency for the downstream and upstream transmission. On the other hand, the XG-PON is newer standard of the passive optical network with the new frame structure. The authors do not compare the initial process in the GPON and XG-PON networks.

The work [1] dealt with the TC layer for the XG-PON networks. We explained the collision issues in a registration process, the influence on the equalization delay on initialization process, and the influence of a refractive index on the RTD (Round-Trip Delay) in the XG-PON networks. In general, we did not deal with the initial process of the ONU when first connecting into the PON.

The paper [4] analyse security issues of the GPON networks. We focused on the security because the GPON networks have security issues with the key establishment. In this establishment key or parameters for the key computing are transferred as a plain text. In general, the GTC (GPON Transmission Convergence) frame has a complicated structure and it is not quite easy to decode the frame. However, there are some companies which can provide an analyser for GPON and XG-PON networks. That is the reason why it is necessary to solve these security issues.

Further, the paper [5] explored redesign of Metro-Access Next Generation PON. They reached $100 \mathrm{~km}$ with redesigning of the TC layer. However, the authors did not assume the different propagation delay because OLT is able to correct the various length of the ODN by an equalization delay. More precisely, the equalization delay is considered up to $60 \mathrm{~km}$ of ODN.

The article [6] provided stability and delay analysis of the EPON registration protocol. They used the Markov model and
Discrete-Time Markov Chain for the registration process simulation. The authors verified the influence of the registration delay using simulation model. Further, the EPON uses the different registration process in comparison with GPON.

The work [7] compared the economic aspects between the GPON and EPON networks. As was mentioned before, EPON is dominating in Asia and GPON is dominating in Europe. They consider 100000 customers in ISP's network and compared which standard provides the higher bandwidth to each customer. The GPON standard is more efficient when the customer wants to have a higher bandwidth (with lower amount of OLTs the GPON standard is able to provide higher bandwidth that EPON).

Further, the paper [8] introduced capacity and delay analysis of Next-Generation PON. They took stock of the huge PON with different standards (EPON, GPON, and XG-PON) with a numerical and simulation models. These simulations did not consider the initial phase where it is necessary to solve the various delay of each packet. On the other hand, it is not more probable that the one ODN will contain the various types of standards and their upgrades.

The authors [9] dealt with link utilization and comparison of EPON and GPON access network cost. They compared EPON and GPON frame structures and DBA (Dynamic Bandwidth Allocation). The analysis showed that GPON systems uses the link capacity more efficiently than EPON systems [9]. On the other hand, the GPON transceivers are more expensive in comparison with the EPON transceiver.

The goal of this paper is to enhance the initial phase of first ONUs connection into the ODN. We compare the standard algorithm with our novel technique. In other words, the main contribution of this work is a novel technique to establish a connection between OLT and ONU with the same frame structure and keeping a state machine for establishment connection.

\section{ONU ACTIVATION PROCESS}

When the ONU is connected into the PON for the first time, it is necessary to go over the activation states. The first state in activation process is the initial state $(\mathrm{O} 1)$ which is the simple state because the ONU needs to receive 3 GTC frames for downstream synchronization. When the ONU powers up, LOS (Loss of Signal) and LOF (Loss of Frame) are asserted (set to 1). Then the first frame is received, the LOS/LOF are cleared (set to 0 ). In detail, the OLT consecutively sends the downstream frames containing the PSBd (Physical Synchronization Block downstream) and Psync fields and has fix length $(125 \mu \mathrm{s})$. The ONU unit needs to pass over the partial states of the initial phase. First, the ONU has to receive at least two frames with the same Psync value. In other words, the receiving of the frames with the exactly same Psync field is called Hunt state. The frames have to be analysed bit by bit because the ONU is looking for the Psync pattern. Once a correct Psync pattern is found, the ONU transition into the Pre-sync state and sets a counter $(\mathrm{N})$ to value 1 [10]. The ONU continues with inspection of each of all receiving frames but with the following frames is the counter $\mathrm{N}$ incremented. It 
is necessary to define the parameter $M$ because it represents the number of renewal. In other words, when $M$ is set to 5 , it means the ONU needs to receive 5 frames with the correct (exactly same) Psync field. On the other hand, when the single frame contains different value in Psync pattern, the ONU moves back to the Hunt state. Then the exact value of frames defines with $M$ are received, the ONU moves to Sync state which is the final state of the Initial state with the correct pass. Note, if the parameter $M$ is set, for example, to 3 instead of 5 (the minimum value is 2), ONU reaches the Sync state earlier but if the ONU does not receive 3 correct frames it moves back to a Hunt state. The ONU moves to the Standby state $(\mathrm{O} 2)$ [10]. Nevertheless, the ONU has been synchronized but the ONU does not know the network parameters (delimiter value, power level, and pre-assigned delay). In other word, Upstream Overhead message has to be transmitted three times by OLT. After that, the ONU configures the parameters and moves to Serial Number state (O3). The OLT sends the serial number request to ONU. The ONU answers with serial number ONU PLOAM (Physical Layer Operation, Admission, and Maintenance) message and waits to answer with Assign_ONU-ID PLOAM message which is sending three times by the OLT. The Assign_ONU-ID message sets the ONU-ID for direct addressing in the network and has to be received before the timer TO1 expires ( 10 seconds). After processing, the ONU with assigned ONU-ID moves to Ranging state (O4). The Ranging state has the most important function, since the ONUs are located in a various lengths from the OLT it is necessary to compute an equalization delay separately for each ONU. In other words, the equalization delay eliminates the various distances between ONUs and OLT with keeping the synchronization of the time slots for the ONUs. The control unit can calculate time equalization delay by the following equation (1) [10]:

$$
\begin{aligned}
& \text { Teqd }=T_{1490, i}+\text { RspTime }_{i}+E q D_{i}+T_{1310, i} \\
& =T_{1490, i} \frac{n_{1310}+n_{1490}}{n_{1490}}+\text { RspTime }_{i}+E q D_{i}
\end{aligned}
$$

where RspTime is the response time ( $\mu \mathrm{s}), E q D_{i}$ is the estimation (pre-assigned delay by OLT) of the equalization delay for the ODN length, $n_{1310}$ represents the group velocity refractive index for $1310 \mathrm{~nm}$ in the ODN, $n_{1490}$ represents the group velocity refractive index for $1490 \mathrm{~nm}$ in the ODN. From the fraction with group velocities the index correction factor can be called. It can be expressed as (2) [10]:

$$
T_{1490, i}=\left(\text { Teqd }- \text { RspTime }_{i}-E q D_{i}\right) \frac{n_{1490}}{n_{1310}+n_{1490}}
$$

Substituting into the expression for the receive instance of GTC frame N we obtain the equation (3) [10]:

$$
\operatorname{Trecv}_{N, i}=\operatorname{Tsend}_{N, i}+T_{1490, i}
$$

The full formula of eq. (4) is [10]:

$$
\begin{aligned}
& \operatorname{Trecv}_{N, i}=\text { Tsend }_{N}+\operatorname{Teqd}\left[\frac{n_{1490}}{n_{1310}+n_{1490}}\right]_{O L T}- \\
& \left(E_{i} D_{i}+\operatorname{RspTime}_{i}\right)\left[\frac{n_{1490}}{n_{1310}+n_{1490}}\right]_{O N U}
\end{aligned}
$$

The ONU sends Serial_Number_ONU message to OLT and waits for an answer. The answer is the Ranging Time message

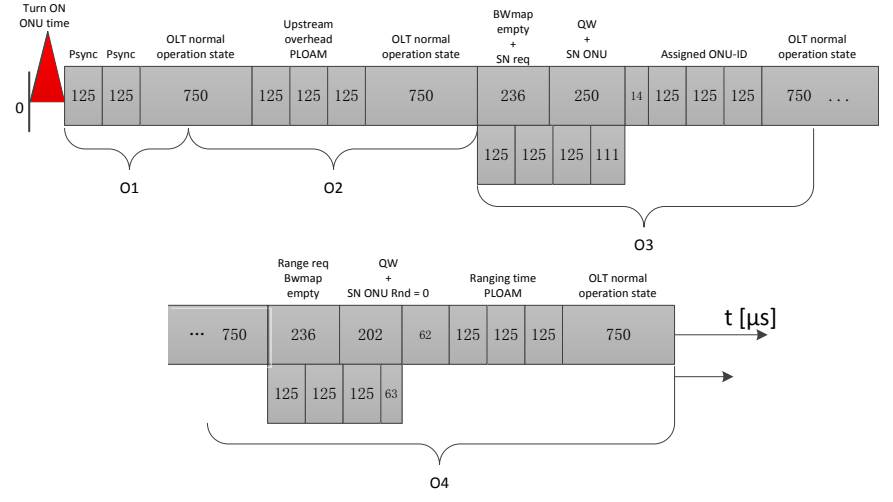

Fig. 2. Activation process based on current algorithm.

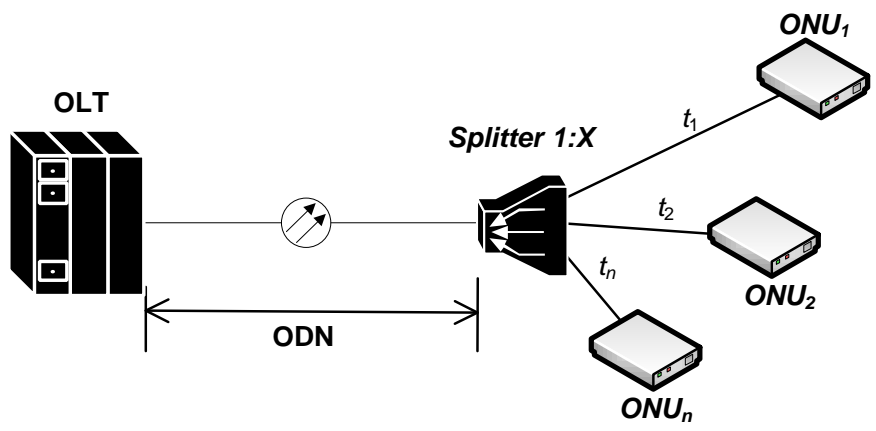

Fig. 3. The simulation topology implemented in Matlab.

with re-calculates the equalization delay (the ONU has only pre-assigned delay) which is set up by ONU. Then, the ONU moves to Operational state (O5) which is the last state for the bidirectional communication. A sequence of the frames for each state is shown in Fig. 2.

\section{SIMULATION SET UP}

We used the Matlab (without Simulink ${ }^{\circledR}$ ) software with own implementation of GPON network (by m-file functions), especially the TC layer model. The physical layer is represented by the length of an optical fibre in order to evolve a velocity of propagation and various split ratios (from 1:16 to $1: 128$ ). The attenuation class B was selected for our simulation model. More precisely, we did not consider the higher split ratio than $1: 128$. In general, for higher split ratios the specification is not allowed [10] and the total attenuation specified for Class $\mathrm{B}$ is not observed. Our GPON simulation model contains the OLT, ONUs, and ODN. We consider the longest ODN for the simple simulation topology which is shown in Fig. 3.

The simulation models were created for the current and the novel algorithms. The following text describes the current algorithm. The variable nONU represents the value of ONUs after the splitter from 2 to 128 values. The parameter MaxDistance provides the distance of ODN. In general, when we define $20(\mathrm{~km})$, it means that our model generates random length for each nONU variable which is save for the equalization delay calculation. Constant $f d$ means the frame duration $(125 \mu \mathrm{s})$ and delay variable adds a time when the counter curTime overflows timer TO1. As was mentioned 
before, only single ONU can be activated after the receiving of Serial Number request because ONUs wait for locally random delay $(0-48 \mu \mathrm{s})$ with answer in the real network. The OLT answers to the first receiving Serial Number ONU message. Our simulation model has no pre-assigned delay and the parameter $M$ is set to 2 . In the Initial phase (O1), Hunt state requires only two frames with the correct Psync field to enter Standby state $(\mathrm{O} 2)$. It is an optional parameter, the ISP can set up this parameter by their consideration. The current flow shown in Fig. 2 repeats each second.

We proposed the novel algorithm for activation process which reduces activation time. As first, there is a generation of array of n-ONUs at random distances from OLT up to $20 \mathrm{~km}$. Then the array is sorted in ascending order due to the distance (this is done because in the best case the closer ONU will respond in the shortest time and the OLT will choose ONU that sends an Assign ONU-ID message first). After that there is a loop for all ONUs which calculates the delay of connecting ONUs to the network. For the first ONU it is:

- $21 \times f_{d}$ - where ONU synchronizing in the upstream direction received Upstream Overhead PLOAM to set the network parameters and responds to Serial Number ONU message.

- $16 \times f_{d}-$ to assign ONU-ID, responds to Ranging Request with Serial Number ONU and receives Ranging Time PLOAM to set equalization delay.

- $3 \times f_{d}-$ when ONU enters Operational State (O5). This delay is optional and is used only for OLT to let ONU be ready for transmission

The total time is $40 \times f_{d}(5 \mathrm{~ms})$ to connect the first ONU to the network. The OLT then sends another Assign ONUID message after 403 frames $(50.375 \mathrm{~ms})$ then the next closest ONU is connected in $403 \times f_{d}+3 \times f_{d}$ (52.750ms). This repeats 19 times until the OLT is able to connect 20 ONUs in 1 second. Note that there is a 2 -frame space between the last ONU in this cycle and the first ONU in the next second cycle so the joining of the ONU at the beginning of each second is delayed by $250 \mu \mathrm{s}$. The OLT is able to connect 128 ONUs in approximately 7 seconds so this setup should be useful when the OLT is rebooted. After that it can operate in normal state.

Nowadays, ISPs use activation process which is defined in [10] but manufacturers can change some unspecified fields of messages because they are undefined. It is only recomendatation which causes the compatibility issues. Our proposed algorithm should be implemented independent (because we use exactly same messages type and format). More details about our proposed algorithm are described in Fig. 4. The initial phase is same as current algorithm defined in [10] but the main disadvantage is smaller number of total register ONUs in the same time. Note that many parameters such as Preassigned delay and ONU-ID are transferred in broadcast mode. The ONU processes all downstream frames while looking for own serial number or ONU-ID if is already assigned. The OLT should operate normally for at least $750 \mu$ s when it transmits by broadcast Upstream Overhead and Assigned ONU-ID PLOAM messages as recommended in [10]. In our implementation we consider that the OLT waits exactly $750 \mu \mathrm{s}$ before transmitting another PLOAM message(s) but vendors may set longer delay which will cause slower activation of ONU(s). We also consider transferring of exactly same parameters but with more grants for ONU at the same time. The main goal is to speed up an activation process after blackout scenario because each ONU needs to be registered by OLT again. Blackout causes that customers are not able to use paid services until their ONU is activated again. For example, when we consider 1024 ONUs, up to tens of minutes or units of hours is needed but with our algorithm the registration process of the same amount of ONUs can be reduced up to units of minutes.

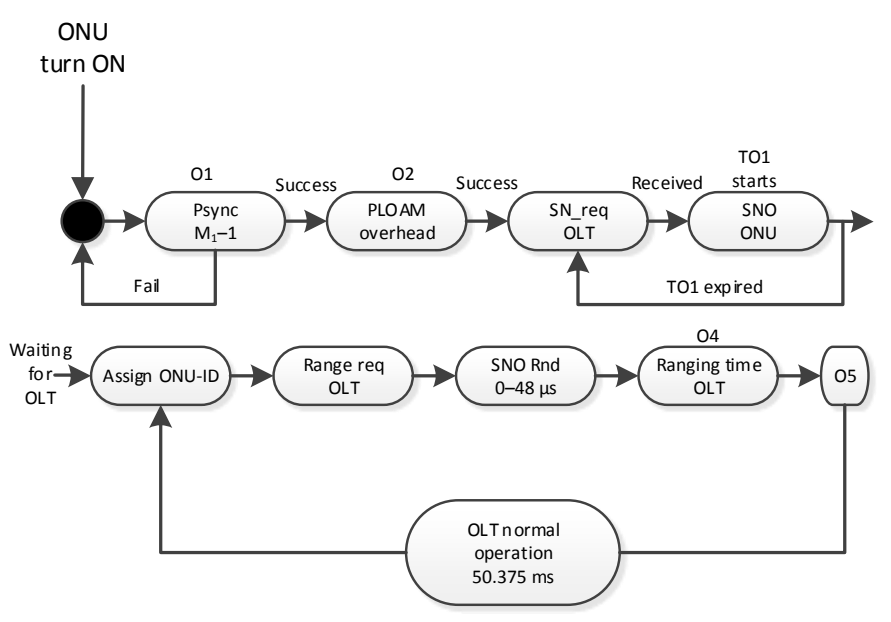

Fig. 4. State machine of proposed algorithm.

The simulation models had been divided into two scenarios, with the current and novel algorithm. The results were disposed by split ratio with the same algorithm. In general, our model had the TC layer for ONU and OLT, optical fiber (represented the velocity of propagation delay - calculated from $c$ and refractive index $n$ ), and length was generated from the range $(1-20 \mathrm{~km})$. The simulation results with current algorithm for split ratios (1:16 and 1:32) are shown in Fig. 5.

In Fig. 5 dependence of split ratio on activation time proposed by our implementation in Matlab is depicted. We did not change state machine and messages format defined in [10]. In general, the current algorithm is able to register single ONU in one algorithm cycle. In the worth case, the last ONU in blackout scenario was connected approximately after 33 seconds. On the other hand, the real networks never contain only $32 \mathrm{ONU}$ at single OLT ports due to the economical aspect of network. The second scenario was for the higher split ratios (1:64 and 1:128) which had the results depicted in Fig. 6.

In the second scenario the results reached the higher values. When we consider the standard OLT with 4,8 or 16 ports to splitters and ODN that means eight times higher total time for the last ONU (applies for the OLT with single Central Processor Unit). In other words, our results need to be multiplied by ports number of OLT. In the best case, the last ONU will be connected after $500 \mathrm{~s}\left(4 \times f_{d}=500 \mathrm{~s}\right)$ or $2000 \mathrm{~s}$ in the worth case $\left(16 \times f_{d}=2000 \mathrm{~s}\right)$. That is the reason why we proposed the novel algorithm for activation process with keeping the frame structure and state machine. 


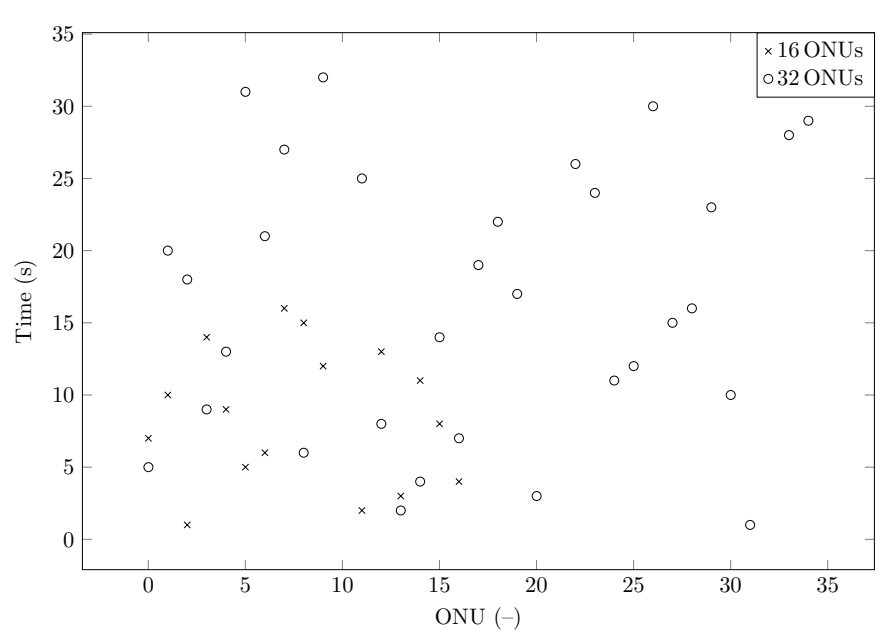

Fig. 5. Activation process for $16 / 32$ ONUs in blackout scenario using algorithm defined in [10] (without changes).

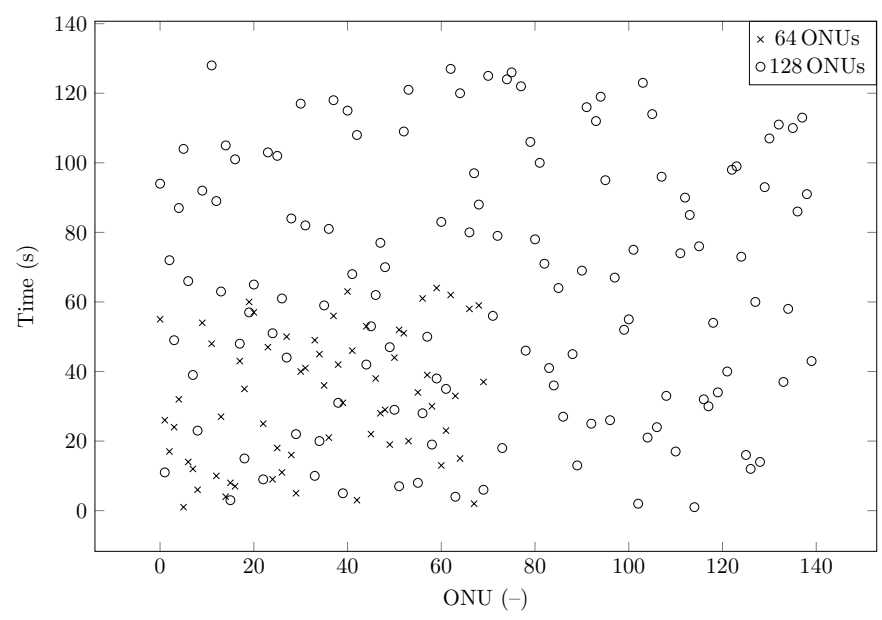

Fig. 6. Activation process for 64/128 ONUs in blackout scenario using algorithm defined in [10] (without changes).

We simulated the same scenarios with our proposed algorithm. At first, Fig. 7 depicted the results for the lower split ratios (1:16 and 1:32).

We reached approximately seventeen times lower time in comparison with the current algorithm. The last ONU will be connected after $\sim 2$ seconds when we talk about 32 ONUs with the same frame structure and state machine. The proposed algorithm does not require any special hardware because we modified a quite window. The quite windows are normally used in the current algorithm (see Fig. 2). The OLT with the quite window has time to process answer to ONUs and should prepare the following messages. The second scenario with higher split ratios (1:64 and 1:128) provided more interested results.

The last simulation of activation process is shown in Fig. 8 . The time $\sim 7 \mathrm{~s}$ for the last ONU is shown in Fig. 8 . It is not the final time because we consider singe port used at OLT but we are able to estimate time for the best and worth case. In the best case, the OLT has 4 ports. Each of them has 128 ONUs (in other words split ratio is $1: 128)$. In worth case we obtained $\sim 28 \mathrm{~s}$ for one port and $\sim 112 \mathrm{~s}$ for 4 ports, respectively. In

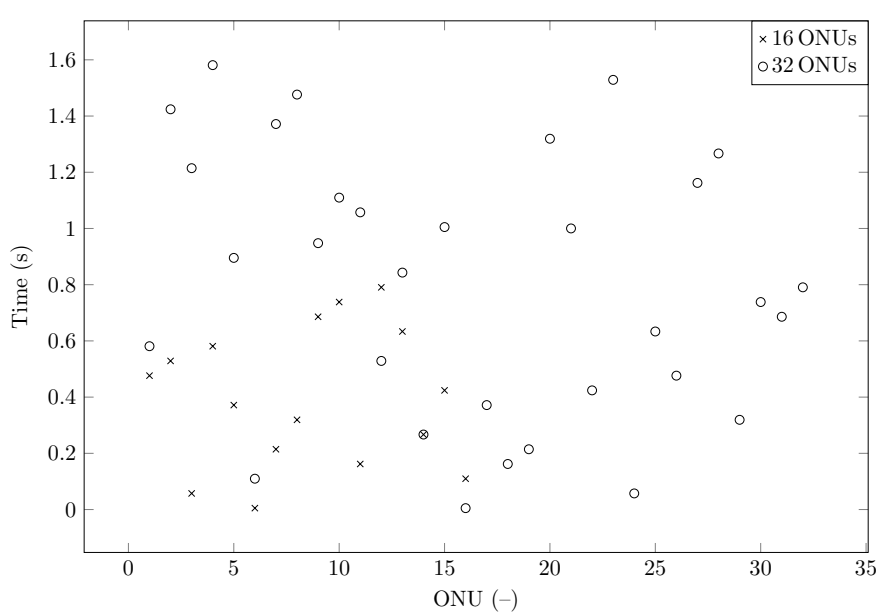

Fig. 7. Time of activation process for $16 / 32$ ONUs in blackout scenario using our algorithm (with changes).

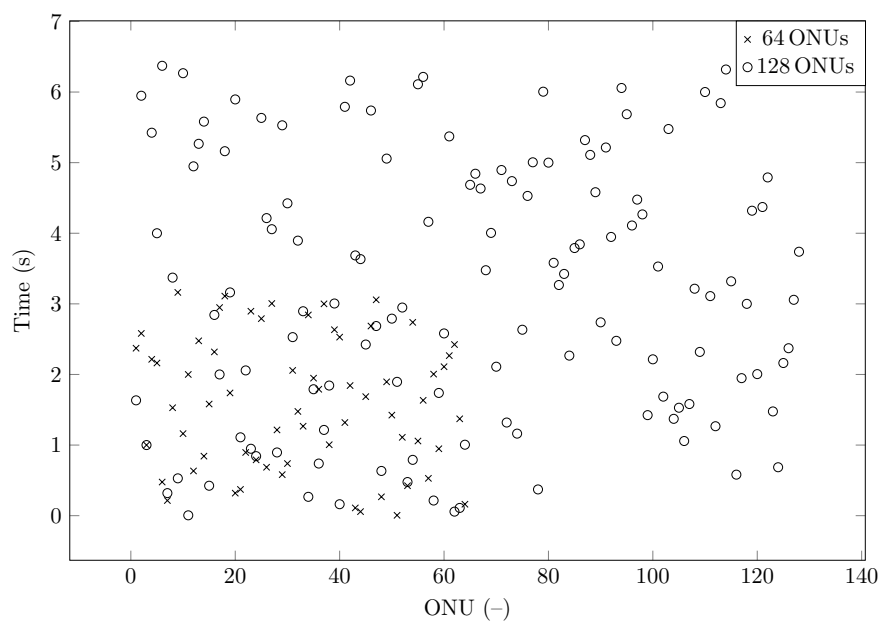

Fig. 8. Time of activation process for $64 / 128$ ONUs in blackout scenario using our algorithm (with changes).

comparison with the current algorithm we decreased nineteen times the total time in activation process. For example, when the OLT has issue and needs to be replaced, with our algorithm the subscribers have a connection time from the seconds to minutes compared to the current algorithm where it takes from tens of seconds to tens of minutes.

\section{CONCLUSion}

This paper deals with novel activation process in GPON. A modification of the current algorithm causes that we are able to minimize the time requirements. For the business or VIP clients it is necessary to serve the best SLA (Service Layer Agreement) at ODN. Therefore, the current algorithm is not able to assure the expeditious reaction. We proposed the novel algorithm which reduces nineteen times the total time of activation process for last ONU in ODN. In other words, we are able to connect more ONUs at the same time. Using of proposed algorithm can be implemented in GPON and XGPON only with a modification of the sequence of the frames.

In future work, we would like to implement our solution into real OLT and analyse the performance of the proposed 
solution.

\section{REFERENCES}

[1] Lukas Koci, Tomas Horvath, Petr Munster, Michal Jurcik, and Miloslav Filka.Transmission Convergence Layer in XG-PON, 2015 38th International Conference on Telecommunications and Signal Processing (TSP), no. $1,2015$.

[2] International Telecommunication Union. G.984.2: Gigabit-capable Passive Optical Networks (G-PON): Physical Media Dependent (PMD) layer specification. 2003. 2015-08-21.

[3] Yuanqiu Luo, Frank Effenberger, and Bo Gao. Transmission convergence layer framing in XG-PON1, 2009 IEEE Sarnoff Symposium, no. 1, pp. $1-5,2009$

[4] Lukas Malina, Petr Munster, Jan Hajny, and Tomas Horvath. Towards Secure Gigabit Passive Optical Networks, In Proceedings of SECRYPT 2015, no. 1, 2015.

[5] Eduardo Tommy Lopez, Victor Polo, J. A. Lazaro, and Josep Prat. Layer 2 redesign for Metro-Access next generation PON, 2014 16th International Conference on Transparent Optical Networks (ICTON), no. 1, pp. 1-4, 2014.

[6] Qingpei Cui, Tong Ye, Tony T. Lee, Wei Guo, and Weisheng Hu. Stability and Delay Analysis of EPON Registration Protocol, IEEE Transactions on Communications, vol. 62, issue 7, pp. 2478-2493, 2014.

[7] Bonilla, Felipe Rudge Barbosa, and Moschim. Techno-economical comparison between GPON and EPON networks, Innovations for Digital Inclusions, 2009. K-IDI 2009. ITU-T Kaleidoscope:, no. 1, pp. 1-5, 2009.

[8] Frank Aurzada, Michael Scheutzow, Martin Reisslein, Navid Ghazisaidi, and Martin Maier. Capacity and Delay Analysis of Next-Generation Passive Optical Networks (NG-PONs), IEEE Transactions on Communications, vol. 59, issue 5, pp. 1378-1388, 2011.

[9] Sami Lallukka, and Pertti Raatikainen. Link utilization and comparison of EPON and GPON access network cost, GLOBECOM '05. IEEE Global Telecommunications Conference, 2005, no. 1, 5 pp.-, 2005.

[10] International Telecommunication Union. G.984.3 : Gigabit-capable passive optical networks (G-PON): Transmission convergence layer specification. 2014. 2015-08-23.

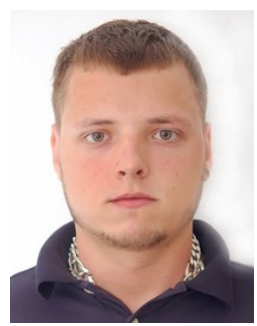

Tomas Horvath (MSc) was born in Havirov, Czech Republic on March 7, 1989. He received his M.Sc degrees in Telecommunications from the Brno University of Technology, Brno, in 2013. His research interests include passive optical networks (xPON), optoelectronics, and BitTorrent protocol. Currently, he has been actually post graduate student at Brno University of Technology, Department of Telecommunications and his topic of dissertation thesis is Optimization services in FTTx optical access networks.

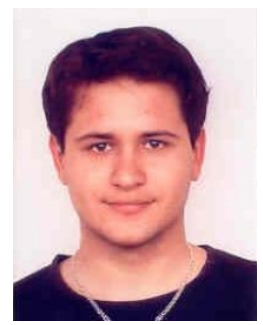

Petr Munster (MSc, PhD.) was born in 1984, in Zln (Czech Republic). He received his $\mathrm{PhD}$ at the Brno University of Technology, Department of Telecommunications in 2014 on the thesis entitled Parameters of the FTTx networks. His current research themes focus on fiber-optic sensors, especially distributed fiber-optic sensors, and also on fiber-optic telecommunications. He has about 50 scientific publications in journals and conferences in last 5 years.

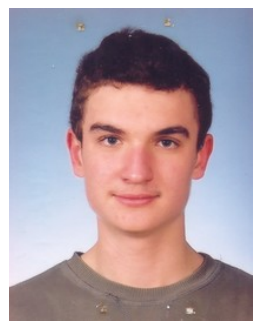

Michal Jurcik (MSc) was born in Zlin on 29 of March 1988. He is a Ph.D. student on the Faculty of Electrical Engineering and Communication Brno University of Technology on the Department of physics which started in 2014. In parallel he is working as software test engineer for Honeywell International. $\mathrm{He}$ is also a co-investigator in project Characterization of materials and advanced coatings in CEITEC. His dissertation is focused on study of cold field emission cathodes and its properties. Due to his dissertation he cooperates with Delong Instruments on electron microscopy. Currently he is working on prototype for cathode etching to improve the quality of etched tips and also programming software for it. He has experiences with programming languages such as $\mathrm{C \#}$ $\mathrm{C}++, \mathrm{C}, \mathrm{VB}, \mathrm{JAVA}$ and Matlab. Biography text here.

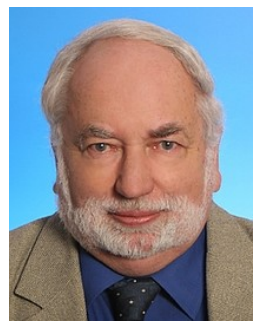

Miloslav Filka (prof.) was born in 1946 in Brno, Czech Republic. Since 2010 he is a professor at the Department of Telecommunications at Brno University of Technology. Hi is a leader of the optical group OptoLab and also head of the Laboratory of transmission media and optical networks. He is a member of a several institutes (e.g. Institute of Electrical \& Electronics Engineeres) and is also committee of many conferences (International Conference Telecommunications and Signal Processing, International Conference New Information and Multimedia Technologies). His current research themes focus on fiber-optic telecommunications, especially FTTx technologies. Biography text here. 